\title{
La violence quotidienne des enfants de la rue : Bourreaux et victimes à Lubumbashi
}

\section{Olivier Kahola Tabu}

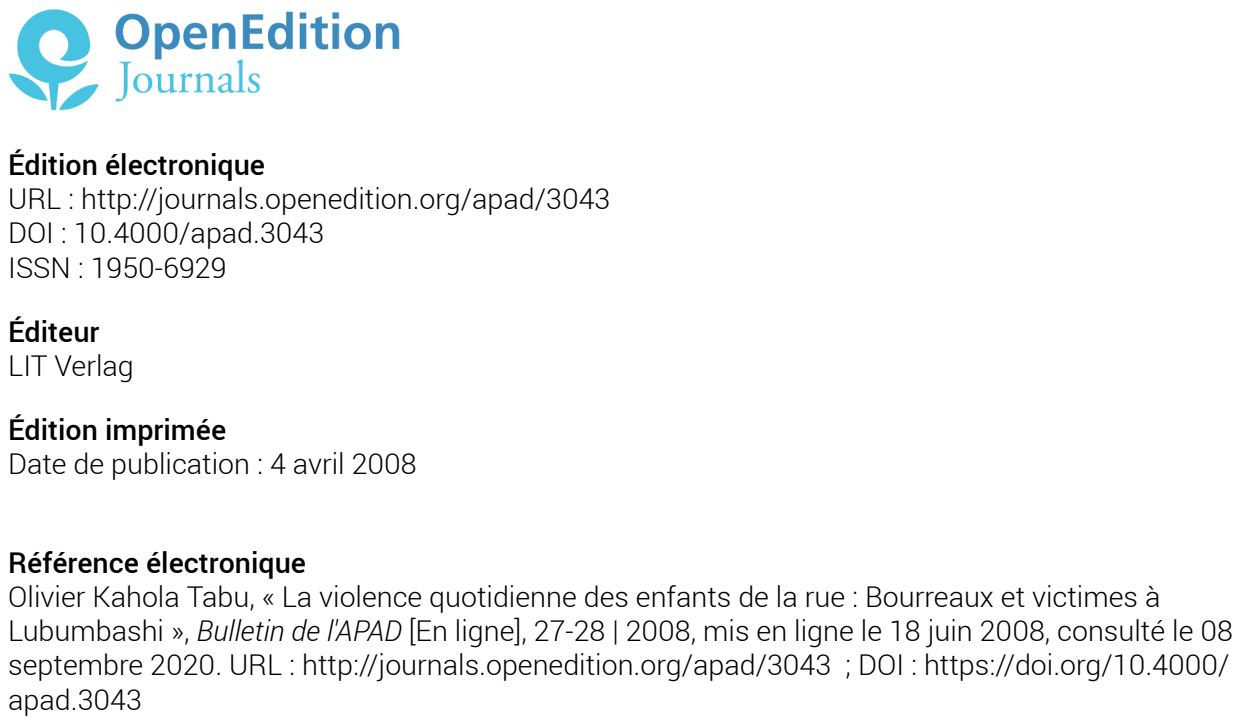

Édition électronique

URL : http://journals.openedition.org/apad/3043

DOI : 10.4000/apad.3043

ISSN : 1950-6929

Éditeur

LIT Verlag

Édition imprimée

Date de publication : 4 avril 2008

Référence électronique

Olivier Kahola Tabu, «La violence quotidienne des enfants de la rue: Bourreaux et victimes à Lubumbashi », Bulletin de l'APAD [En ligne], 27-28 | 2008, mis en ligne le 18 juin 2008, consulté le 08 septembre 2020. URL : http://journals.openedition.org/apad/3043 ; DOI : https://doi.org/10.4000/ apad.3043

Ce document a été généré automatiquement le 8 septembre 2020.

Bulletin de l'APAD 


\title{
La violence quotidienne des enfants de la rue : Bourreaux et victimes à Lubumbashi
}

\author{
Olivier Kahola Tabu
}

Vivre violenté à la maison ou aller dans la rue?

1 Le problème est de comprendre comment les enfants en arrivent là. Qu'est-ce qui est si insupportable dans la vie familiale qu'on y préfère la violence quotidienne des interactions dans la rue? Le phénomène social des "enfant de la rue " témoigne manifestement de graves dysfonctionnements du lien social et de l'urbanité dans beaucoup de pays en voie de développement. Il apparaît en premier lieu que nombre de ces enfants ont fuit leurs familles suite à la dégradation des conditions de vie familiales, ainsi qu'en témoigne le cas de Jeannette :

«Je menais une bonne vie à la maison avec mes parents. À la mort de mon père, la vie a profondément changé dans la maison. Manger devenait un casse-tête. Finalement ma mère s'était remariée. Malheureusement pour moi, mon parâtre ne voulait pas de ma présence à la maison. Ma mère pour préserver son mariage m'avait envoyé chez ma grand-mère. Quelques mois après, ma grand-mère est morte du choléra. Je devais rentrer chez ma mère malgré l'antipathie de mon parâtre. Ma présence dans la maison n'arrangeait pas mon parâtre. Un jour ma mère m'avait confié la clé de la maison pour des travaux ménagers. En faisant la vaisselle, par mégarde, j'avais cassé une assiette en porcelaine. Mon parâtre qui était là, mécontent que j'aie cassé son assiette, s'était rué sur moi. Il m'avait fortement giflé et je m'étais cogné la tête contre le mur et je saignais du nez. Dans sa colère, il ne s'était pas aperçu que je saignais et il a continué à me battre, jusqu'à ce que je me sois sauvée. Quand ma mère est rentrée, elle m'a trouvé avec une figure déformée. Je lui ai relaté ce qui s'était passé. Mais, grande a été ma surprise de voir ma mère s'en prendre elle aussi à moi. Elle m'insultait pour plaire à son mari. Finalement, dans cette maison, ma vie était devenu un enfer. À chaque lever du soleil, je savais que mon parâtre m'insulterait ou me frapperait sans cause. Je me plaignais auprès de ma maman, mais cette dernière ne me soutenait guère. Un autre jour, mon parâtre avait égaré une somme de 5000 francs congolais dans la maison. Pour mon parâtre, je m'étais infiltré dans sa chambre et j'avais volé son argent. Avant même qu'il ne m'entende, il m'avait étranglé et je ne pouvais ni crier 
ni bouger. Heureusement pour moi, J'ai eu la vie sauve grâce à papa Gilbert qui était intervenu. Mon parâtre furieux sous l'emprise de la colère bégayait et m'insultait : Adultérine, je ne veux plus te voir dans ma maison. Ma mère impuissante devant ce qui se passait s'est ralliée à la cause de son mari en me promettant de me brûler les mains. J'ais alors compris que ma place n'était plus dans cette maison et que je devrais aller vivre dans la rue. C'était là mon destin ! " (Jeannette, âgée de 13 ans)

2 Le cas de cette fille donne l'image des conditions de vie qu'endurent certains enfants dans leur famille. Au quotidien jeannette était victimisée par son parâtre. Sans doute " Mille et une raisons peuvent être évoquées par les enfants pour expliquer ou justifier leur fugue. Entre raisons objectives et celles subjectives, il existe une plage pouvant donner libre cours de toutes formes d'affabulations. Il arrive même que l'enfant trompe ou qu'il se trompe lui-même sur ce qu'il croit être le vrai motif de sa fugue». (Mulumbwa Mutambwa 2005: 38). Mais Jeannette vivait manifestement dans une situation d'insécurité permanente et de maltraitance traumatisante qui a fini par l'exaspérer. Incapable de vivre dans un cadre familial hostile, elle a cherché des solutions palliatives qui l'ont conduit dans la rue.

3 A Lubumbashi, les familles ne sont pas restreintes au père, à la mère et aux enfants. La question de fond est donc de se demander pourquoi les enfants maltraités ne jouissent pas de la solidarité de leurs proches parents? Parfois, quand l'enfant victime dénonce les mauvais traitements qui lui sont infligés, les parents proches se contentent de le blâmer en le traitant de menteur ou alors, ils l'encouragent à endurer ses souffrances. Ordonez souligne à ce propos que l'enfant de la rue « est un enfant qui a fui sa maison en réaction à un contexte familial violent ou sans capacité de contenance. L'enfant cumule des sentiments d'hostilité durant des années, il ne se sent plus relié à personne. Dans ce climat d'hostilité, ces enfants se résolvent à partir dans la rue, car ils se considèrent comme étant des éléments gênants. Si dès leur départ, les parents ou les membres apparentés ne viennent pas les rechercher, ils consolident leurs liens avec la rue ». (Ordonez, cité par Aguilar $1997: 140$ ).

4 Les conditions d'assujettissement que certains parents imposent aux enfants sous leur responsabilité sont déplorables. Actuellement, la maltraitance de certains enfants est justifiée par le soupçon à la sorcellerie qui fait d'eux la victime désignée de toute lrépression familiale. Jeannette s'est retrouvée dans la rue comme par fatalité. Pourquoi la rue, pourquoi pas ailleurs? L'enfant s'est sentie rejetée par ses proches parents : Sa présence était indésirable. Les voisins assistant à ces violences se défilaient en disant qu'ils n'avaient pas à s'ingérer dans une affaire familiale. Dans l'indifférence absolue de la famille et des voisins, l'enfant se trouve seul devant un choix capital. Rester à la maison et supporter les exactions infligées ou partir loin de ses persécuteurs et risquer d'affronter d'autres violences. La décision de partir a triomphé, car le contexte quotidien est véritablement insupportable.

5 La rue, contrairement à ce que les enfants fugueurs pensent, n'est pas un espace de vie et de gain faciles. Ils doivent prouver leur endurance physique et morale face aux sévices corporels infligés dès leur arrivée par les occupants de la rue. C'est une sorte de rite de passage. Les habitués de la rue qualifient les conditions de vie dans la rue de pima uyambe ou " oses et chies ». Ceci pour dire que dans la rue, règne un potentiel de victimisation multiple qui ne tient pas compte de l'âge ni du sexe. Dans ce contexte, ce n'est pas la morale qui régit l'acte posé, mais plutôt l'objectif de se protéger ou de consolider son identité dans la rue.

Le néophyte et le rite d'intégration 
6 Vue de l'éxtérieur, la rue paraît être ouverte au refuge de tous les enfants. C'est une vision biaisée. Tout enfant qui décide d'élire domicile dans la rue doit accepter d'endurer les atrocités qui y permettent son intégration. Ce rite a un double objectif : Évaluer l'endurance physique du néophyte et adapter l'initié aux rudes conditions qu'il aura à affronter dans la rue. Ainsi, un fugueur qui demeure dans la rue malgré les diverses exactions qu'il y a subi est jugé par les « anciens» apte à y mener sa vie. A ce niveau, l'attitude de l'enfant fugueur est complexe. Ayant fui le cadre familial à la suite d'un cumul des mauvais traitements, il se retrouve dans la rue où il est accueilli par la violence des enfants de la rue. Que faire? Regagner la maison après une fugue? L'enfant est souvent bien embarrassé et pour des raisons obscures, il préfère la plupart du temps rester dans la rue tout en ayant le secret espoir qu'un membre de sa famille vienne l'en sortir.

Le cas du rite d'intégration de Xavier :

« Un soir, j'ai fui la maison car ma marâtre me persécutait régulièrement. J'en avais marre et je me suis décidé à aller vivre dans la rue. Ce jour là, je ne savais pas où aller. Rentrer à la maison, c'était exclu pour moi. Je me disais que peut-être mon père viendrait me chercher. Mes attentes ont été vaines. Alors, je me suis rendu au marché Mzee Kabila pour y passer la nuit. Vers 20 heures, je dormais quand un groupe d'enfants m'a réveillé avec brutalité. Sans me demander quoi que ce soit, l'un d'eux m'a fortement giflé et je suis tombé. Avec le concours des autres, il m'a introduit dans un sac et ils commencèrent à me donner des coups de pieds comme s'ils jouaient au football. Je criais, mais personne ne venait à mon secours. J'ai alors feint de mourir. Je ne bougeais plus. Ils ont cessé de me battre et se sont retirés un moment. L'un d'eux est venu me tirer du sac. Je saignais du nez. Après quelques minutes, je me suis levé et je fuyais de toute ma force. Quand j'ai vu les policiers en patrouille, j'ai accouru vers eux pour chercher protection. Mes bourreaux m'avaient pourchassé et ils demandèrent avec insistance aux policiers de me relâcher. Quand les policiers m'ont demandé d'où je venais et quand je leur ai répondu que j'ai fui la maison à la suite des mauvais traitements de ma marâtre, ils m’ont chassé en disant à mes persécuteurs : "prenez-le ", c'est votre camarade. Je ne savais où aller. Mes persécuteurs m'ont brûlé le corps avec du plastique liquéfié. Quand ils m'ont relâché, je ne pouvais plus marcher. Tout mon corps était douloureux. Rentrer à la maison? Je rencontrerais ma marâtre et elle me torturera de nouveau. Je me suis alors résigné à mourir dans la rue. Le jour suivant quand mes persécuteurs m'ont revu, ils étaient étonnés que je ne sois pas rentré à la maison après tout ce qu'ils m'avaient fait subir la nuit précédente. Le chef de la bande m'a tendu la main en me disant que je pouvais vivre dans la rue car j'avais un cœur dur. Il m'a associé à son groupe et depuis lors, je vis dans la rue ».

8 Gisèle quant à elle, témoigne ainsi :

« Il faut avoir un «gros cœur» pour vivre dans la rue. Quand je « revis » ce qu'on m'a fait subir pour mon intégration dans la rue... Jamais, je ne pardonnerai à ma marâtre. C'était un dimanche quand je me suis résolue de quitter la maison de mon père après que ma marâtre m'aie brûlé les mains avec de l'eau bouillante. Alors, je me suis retrouvée vers 21 heures au marché Mimbulu. Me tordant de douleur à cause de la brûlure, je ne savais pas où aller et un groupe de filles de la rue m'a recueilli. Elles me sermonnaient en ces termes : «tu viens nous rivaliser ? Tu penses que c'est facile de vivre dans la rue? Tu aurais mieux fait de rester à la maison malgré les persécutions. Ici, tu vas vivre des choses pires que ce que tu as vécu à la maison » : Elles se sont alors ruées sur moi et elles m'ont déshabillé. J'étais toute nue. Elles ricanaient en me voyant dans cet état. La plus grande de la bande m'intima l'ordre de me taire, menaçant que si j'osais crier, elle m'enfoncerait un bâton dans le vagin. Je tremblais et je pleurais. Elles m'ont fait des attouchements sexuels. Puis, elle a demandé à Véronique d'appeler deux garçons de la rue. 
Quelques minutes plus tard, véronique est revenue accompagnée de deux garçons. Les filles m'ont immobilisé les bras et jambes. La fille chef de la bande a demandé aux garçons de me violer à tour de rôle. C'est le jour où j'ai connu l'homme pour la première fois. Le premier m'a défloré et le second n'a fait qu'accentuer ma peine. Quand je criais, la fille chef de la bande m'étranglait ou me giflait de toutes ses forces. Par la suite, elle m'a dit que maintenant j'étais devenu une grande fille et je n'aurai plus peur des garçons. Avant qu'elle ne me laisse, elle m'a forcé à fumer du chanvre et je me suis exécutée craignant de subir de nouvelles violences sexuelles ».

Ces témoignages montrent les violences que les enfants de la rue font subir aux néophytes. Ces violences en forme de rite d'intégration se passent la nuit car c'est à ce moment que les habitués de la rue peuvent constater l'arrivée de nouveaux fugueurs. Une fois repérés, ils leur infligent des tortures inimaginables dans une indifférence sociale totale. Les garçons sont systématiquement lynchés, lapidés, tailladés à coups de rasoir, brûlés avec du plastique fondu, ligotés et jetés dans la fosse. Parfois, le corps des enfants tailladés au rasoir est enduit de sel ou de piment. Quant aux initiés chanceux, ils sont simplement fouettés ou battus à coups de poings. La scène d'un rite d'intégration dans le monde de la rue est désolante. Les "anciens» de la rue, sous l'effet de la drogue, se montrent sans pitié pour les nouveaux venus. L'« initié » crie de toutes ses forces pour appeler un éventuel secours. Vains sont ses efforts, car personne ne se hasarde à lui porter secours. Pas même un policier ne peut oser une telle entreprise. Dans ce processus d'initiation "rituelle », la situation paraît normale pour les initiateurs. Impassibles, ils s'encouragent dans leur langage argotique. Les bourreaux disent au néophyte :

"C'est ça la rue, tu seras couramment battu et il te faut beaucoup d'endurance pour survivre dans cette vie. Personne ne viendra défendre ta cause, alors il faut savoir encaisser quand il le faut et riposter si tu en as l'opportunité. Quand tu voleras, on te battra, alors, il faut que tu sois immunisé contre la douleur ».

10 En ce qui concerne les filles, la plupart des sévices qui leur sont infligés sont sexuels. Dénudées, humiliées et violées, tel est le sort qui leur est réservé. Mais elles sont aussi torturées, il arrive que les habituées leur brûlent les petites lèvres du vagin à l'aide d'une cigarette. Elles peuvent aussi avoir recours aux attouchements sexuels violents. Ces pratiques ne tiennent pas compte de l'âge de l'« initiée ». D'une manière générale, elles pensent que c'est la bonne manière d'apprendre à l'initiée, quel que soit son âge, à ne plus avoir peur des hommes : Une fille qui a été violée n'aurait plus jamais peur des hommes. Elles pensent ainsi, les préparer à survivre dans la rue, car les filles ne peuvent pas exercer les mêmes petits métiers que les garçons, il convient alors les préparer à la prostitution par la «désacralisation" de l'homme et de son sexe. Il importe de souligner que dans ce rite d'intégration, les néophytes filles ne sont pas exclusivement violentées par les anciennes occupantes de la rue. Il arrive qu'elles soient recueillies par les garçons de la rue qui les violent en groupe ${ }^{1}$. Ces violences corporelles et sexuelles ont lieu la nuit, dans l'indifférence totale d'une société confinée derrière les murs des espaces privés. Le jour, les passants remarquent simplement que les enfants de la rue ont des cicatrices et portent des blessures, mais ils n'imaginent pas que ces marques sont le résultat des sévices subis dans l'anonymat de la nuit.

Violences entre passants et enfants de la rue

11 Quand on observe les comportements des passants on constate une certaine méfiance et même une hostilité a priori vis-à-vis des enfants de la rue. Cette attitude des passants est aparemment le reflet de l'image qu'ils ont des enfants de la rue qui sont identifiés comme agressifs et brutaux. De fait, en situation de survie, les enfants de la rue 
développent des sentiments violents. Ils ne se gênent pas pour jeter des pierres aux gens qui les empêcherait de voler un passant. Et, quand ils se bagarrent entre eux, ils utilisent des armes blanches. Ces attitudes cristallisent la méfiance et l'hostilité des passants.

Dans la trilogie (rue-enfant-passant de la rue) les rapports sont ambivalents. Les enfants de la rue estiment que la rue, c'est leur domaine. Toute personne qui l'investit doit se soumettre à sa règle : Le rapport de force violent du vol et des humiliations. Quand les enfants de la rue «filent » un passant pour lui soutirer de l'argent dans son sac, il vaut mieux ne pas intervenir. Celui qui s'y hasarderait serait pourchassé par les enfants au risque de se faire lapider ou taillader à coups de rasoir. Les passants connaissant l'agressivité de ces enfants ne veulent pas qu'ils s'approchent de leur terrain d'activité. Pour éviter tout ces problèmes, les passants ont développé un réflexe d'hostilité a priori envers les enfants de la rue. Ainsi, quand un enfant de la rue mendie, le passant ne réagit pas à ses sollicitations. On ne lui accorde ni regard, ni écoute. Si l'enfant de la rue continue à harceler, on l'insulte ou on l'écarte d'un revers de la main. Alors, certains enfants réagissent par l'insulte même quand le passant est passif.

13 Ainsi, l'interaction entre les enfants de la rue et les passants est incertaine, les deux parties se sentent vulnérables et en insécurité. L'enfant est prédisposé à recevoir des coups à chacune de ces tentatives de survie, tandis que Le passant doit rester concentré pour éviter de se faire voler son argent ou ses biens tout en évitant de se faire insulter. Dans cet univers de méfiance agressive, s'il arrive qu'un passant victime d'un forfait de la part d'un enfant de la rue attrape le fautif, il se fait justice et personne ne peut l'en empêcher. Réciproquement, quand un enfant agresse une personne dans la rue, la plupart des passants restent indifférents selon la formule populaire mubaache baishane ou «laissez-le en finir». Ainsi, dans ces interactions passants-enfants de la rue, la violence est quotidienne. À ce propos, nous rapportons un cas qui s'est passé devant l'hôtel X au centre ville :

«Monsieur W. gare sa voiture dans laquelle il y avait un carton d'argent. Pendant que le monsieur monte dans sa chambre, un enfant de la rue déverrouille la portière, et s'introduit dans la voiture pour y soutirer quelques billets de francs congolais. Mais, il se fait surprendre par le propriétaire qui n'avait pas traîné. Le monsieur s'est saisi de l'enfant, est allé ouvrir le capot du coffre arrière d'où il a tiré un tourne visse pour crever l'œil de l'enfant. L'enfant a crié, mais le monsieur est monté dans sa voiture et a démarré... »

Cet acte de violence dépasse l'entendement et interpelle. La faute commise par l'enfant a été très sévèrement punie par Monsieur $\mathrm{W}$. Et cette réaction violente à la violence de l'enfant ne peut être approuvée en aucune manière. Cependant, les passants ayant assisté à la scène ne sont pas intervenus malgré leur stupéfaction de de ce qui venait d'arriver à l'enfant, laissant le monsieur monter dans sa voiture et s'enfuir. La passivité des passants est une des sources de la violence qui s'installe dans la société et qui, par sa banalisation, s'affirme comme une norme sociale. Avant que le propriétaire du véhicule ne crève l'œil de l'enfant de la rue, certains passants avaient assisté impuissant à l'infraction et à la tentative de vol. Mais, personne n'avait essayé d'arrêter l'enfant. Et pourtant, si l'enfant avait réussi son forfait, Monsieur W. allait se plaindre de son triste sort. Face à une telle complicité entre les passants de la rue (la société ?) et les enfants de la rue, Monsieur W. n'a pas trouvé mieux que de se faire justice lui-même! Les mauvais traitements qu'infligent les enfants de la rue aux passants sont à peine perceptibles. Les principales victimes sont les femmes et les filles. Sur les 
avenues Djamena et Tabora, les enfants de la rue obligent les filles qui passent à leur laisser de l'argent. Si elles n'en ont pas, elles sont menacées d'être lapidées. Parfois, elles se font ravir leurs coiffes. Ces incivilités brutales sont fréquentes et jalonnent le quotidien de notre société. Par leur absence de réaction, les hommes qui assistent à ces scènes de violence ordinaire sur les filles semblent cautionner les pratiques violentes des enfants de la rue.

Ce constat laisse penser que les enfants de la rue et les passants, tour à tour victimes et bourreaux, partagent le même mépris de la personne humaine. L'enfant de la rue est insensible à la douleur que ressent le passant tandis que ce dernier est insensible aux souffrances de l'enfant de la rue qu'il considère comme un rebut de la société. Aurore d'Haeyer évoque ce mépris en ces termes: "un étudiant belge travaillant avec les enfants des rues s'est hasardé à leur offrir de partager son repas. La tenancière du restaurant a jeté la nourriture sur le sol et refusé catégoriquement que les enfants mangent assis sur une chaise. Pour la tenancière, on n'invite pas les déchets à s'attabler » (D'Haeyer $2004: 11$ ).

Exclusion et violence de la société contre les enfants de la rue

La présence des enfants dans la rue donne l'image d'une société qui ne se préoccupe pas du devenir de sa jeunesse. Mais surtout d'une société qui cautionne passivement la victimisation de ces enfants abandonnés. Ces enfants appelés shegue sont clairement exclus de la société. Tous ont le même point commun : l'abandon familial, renforcé par la démission sociale collective.

Une réaction à la relégation sociale

Andrea REA affirme que « les solidarités sociales, communautaires et familiales ne sont même plus à même d'assurer la relève de l'Etat. Face à l'exclusion sociale, la violence des jeunes s'inscrit dans une structure et constitue une réponse à l'exclusion sociale qu'ils subissent. Ceci ne la justifie pas mais permet de comprendre que la violence s'inscrit dans une structure et constitue une réponse à des expériences de la vie. L'exclusion n'est pas en cela qu'une expérience liée à l'absence de travail ou de revenus mais plus généralement à une relégation sociale et politique, c'est-à-dire la non prise en compte de leur position de dominés ». (Rea 1999 : 36). Je pense que c'est dans cette optique qu'il faut comprendre la violence que les enfants infligent à la société. Ainsi, à Lubumbashi, il fut un temps où les enfants de la rue avaient décidé que les filles ne devraient plus porter des pantalons. Celle qui se hasardait à le faire était déshabillée en public sous les applaudissements des passants. Cette situation s'est généralisée à tel point qu'aucune fille n'osait plus porter un pantalon. La violence avec laquelle les enfants de la rue ont traité les filles était surprenante. Les filles étaient pourchassées et celles qui tombaient entre leurs mains étaient déshabillées après avoir été battues. Cette violence particulière bénéficiait d'une certaine caution sociale. Les policiers qui auraient dû protéger les filles, encourageaient tacitement les shegue à violenter les filles et, pendant qu'ils s'acharnaient contre elles, les policiers s'éloignaient davantage du lieu où les filles étaient violentées. La violence des shegue est une réaction à l'indifférence sociale face aux souffrances quotidiennes qu'ils subissent.

Une victimisation exclusive par la société

18 L'enfant de la rue est-il une victime de l'exclusion sociale ? Positive serait d'emblée la réponse, parce que l'enfant de la rue comme le souligne Riccardo Lucchini «est présenté comme la victime d'un environnement exclusivement violent ». (Lucchini 1998 : 358). Cependant, cette perception me semble réductrice au sens où l'exclusion 
sociale que connait l'enfant de la rue me semble s'expliquer avant tout par un comportement violent, asocial voire déviant. Certes, une raison première tient sans doute au fait que la désarticulation socio-économique des familles a mis en cause la solidarité familiale, mais on peut se demander si la violence des enfants fugueurs n'est pas la vraie raison de la dureté avec laquelle le corps social les traite. La question reste ouverte. Néanmoins, les faits témoignent que les enfants vivant dans la rue sont socialement exclus. Les églises qui par charité devraient s'occuper des enfants abandonnés, participent au processus d'exclusion familiale au prétexte que ces enfants seraient des «sorciers" responsables de tous les malheurs qui frappent leur famille. Certains pasteurs après des longues prières tonitruantes accompagnées de transes, prophétisent la sorcellerie des enfants. Alors, les parents « révélés de la sorcellerie » de leurs enfants, les oppriment. Ces enfants sont injustement incriminés pour des crimes qu'ils ignorent et dont ils sont bien sûr innocents. Pour les punir, ils sont privés de nourriture, un jeûne « sec » (sans boire ni manger) de trois jours leur est imposé. Pour les désenvoûter, on les soumet aux prophètes. Ces derniers, avec l'aval des proches parents, imposent des supplices aux enfants pour les exorciser : Ainsi, ils les fouettent ou les tabassent sous prétexte que les sorciers sont têtus et qu'ils n'avouent qu'après avoir été maltraités. Dans un tel contexte, les enfants finissent par prétendre être sorcier, en rapportant des hallucinations. Ce phénomène a pris une telle ampleur que tout enfant de la rue est aujourd'hui qualifié de «sorcier». La relation dyadique d'exclusion des enfants impliquant la famille et l'église est alarmante. Aurore D'Haeyer confirme cette violence qui s'installe dans notre société : " pour exorciser ces gamins, certains pasteurs utilisent la manière forte. Ils font ingérer de puissants vomitifs et laxatifs aux petits afin de les vider des restes de chairs humaines qu'ils disent avoir consommés par la nuit » (D’Haeyer $2004: 40$ ).

Dans ce climat d'accusation et de culpabilité, les enfants ont la conscience chargée. Ils sont traumatisés, car à chaque fois qu'il y a un cas de maladie dans la famille, ils sont menacés de mort si le malade ne recouvre sa santé. Parfois, sans l'avoir cherché, ils se retrouvent jetés à la rue où les attendent d'autres mauvais traitements :

Georgette rapporte le cas de la petite Plamedi âgée de 12 ans :

«Une nuit, nous étions sept filles dans notre Karema. Plamedi était la plus jeune de nous toutes. Quelque temps après, une voiture avait garé devant nous. La vitre descend et un papa m'appelle. J'y vais et il pointe Plamedi. Je fais venir Plamedi. En ma présence, le papa avait demandé si Plamedi pouvait aller avec lui, car il veut l'adopter. J'avais incité Plamedi à accepter la proposition. Elle avait accepté et le papa l'avait embarqué dans sa voiture. C'est après que j'ai su que le papa là était à la recherche d'une fille vierge pour ses charmes magiques. Plamedi avait été amenée dans un hôtel à la Ruashi. Là, le papa avait couché Plamedi par force. Plamedi ne pouvait pas le supporter et elle criait, quand deux policiers de passage survinrent et obligèrent le papa d'ouvrir la porte. Le gérant de l'hôtel, voyant les policiers avait fui. Quand les policiers se sont introduits dans la chambre, Plamedi était presque morte. Pour se tirer de l'affaire, le papa avait proposé une grosse somme d'argent aux policiers. Ces derniers ont empoché l'argent. Se servant de la voiture du papa, ils ont acheminé Plamedi dans un centre de santé de la Ruashi prétextant qu'elle avait été violée par un monsieur et que pendant leur intervention, l'homme avait pris la fuite ".

21 Les filles de la rue subissent le viol dans un silence social absolu. Nous ne réalisons pas l'impact que les viols provoquent dans la vie de ces enfants. Dominique Gauthier souligne que les abus sexuels se situent avant tout dans le domaine très large de 
mauvais traitements infligés aux enfants ${ }^{2}$. La violence sexuelle que l'homme a fait subir à cette jeune fille pubère interpelle les valeurs socioculturelles de notre société.

Ce cas illustre bien ce qui se passe la plupart du temps: Les policiers surprennent l'homme en flagrant délit de viol, mais pour une poignée de billets de banques, ils le soutiennent en maintenant sa culpabilité secrète. Alors, l'enfant victime «se sent trahi dans la confiance aveugle qu'il portait aux adultes, l'abuseur lui renvoyant, par lui-même ou par ses actes, des sentiments de souillure, de honte et d'anormalité. Certains signes peuvent en témoigner: Méfiance vis-à-vis des adultes, irritabilité, agressivité, etc. » (Gauthier 1994). Ce qui est grave dans cette forme de violence est que la plupart du temps, on se contente d'amener la victime à l'hôpital pour y recevoir des soins primaires. Mais psychologiquement, les victimes sont abandonnées à leur triste sort, elles ne bénéficient d'aucun suivi psychothérapeutique d'accompagnement pour leur reconstruction. Or, le véritable enjeu serait de suivre cette enfant humiliée qui désormais se fait une autre image déplorable de l'adulte et de la sexualité. Un autre cas est celui de Annie :

«Alors que vers minuit, j'allais rejoindre mes amies au marché Tunnel, j’ai été arrêtée par trois policiers en patrouille. L'un d'eux m'a interpellée en me demandant d'où je venais et où j'allais à cette heure. Avant même que je ne réponde, un autre m'a intimé l'ordre de les suivre. Quand j'ai trâné les pieds, il m'a giflé en me disant qu'une enfant normale à cette heure-ci, devrait être au lit et à la maison. Il m'a poussé devant eux et il me m'indiquait l'itinéraire à suivre pendant que nous avancions. Chemin faisant, je réalisais qu'ils me conduisaient dans l'enceinte de l'athénée Kiwele. Dans ce complexe, il fait extrêmement noir. Un policier m'a poussé dans une salle de classe et m'a intimé l'ordre de me déshabiller. Sous la menace, je me suis exécutée et il m'a violé. Après, il a fait signe à un autre policier qui s'est introduit dans la salle de classe et qui m'a aussi violé. Quand le dernier est entré, je pleurais et je le suppliais de ne pas me violer car je n'avais plus de force et j'avais des déchirures au sexe. Il m'a pris en pitié et il s'est retiré de la salle de classe en m'abandonnant à mon sort ».

Pour ces cas de viol collectif, Mulumbwa Mutambwa note que « le viol collectif est particulièrement dangereux tant pour la victime que les auteurs. Les violeurs n'ont pas le temps de chercher à porter le condom. La victime risque fort d'être contaminée des IST et VIH/SIDA, de la part de ses bourreaux, qui en retour peuvent également être infectées par le biais de la victime devenue le vecteur commun. Cet acte, du fait d'avoir été commis en groupe semble diluer la responsabilité des auteurs qui peuvent ne pas se sentir totalement responsables du crime. » (Mutambwa 2005 : 26). L'enquête a montré que ces filles se plaignent de coups et blessures. Car en plus du viol qui les exposent à des graves dangers, les filles de la rue sont battues dès qu'elles opposent une résistance à leurs abuseurs. Confrontés à de tels sévices sexuels et corporels, ces enfants de la rue perçoivent la société comme un univers de terreur qui menace leur existence. Il y a plus de vexations que de protections dans le quotidien de leur vie dans la rue. Mêmes ceux qui sont censés les protéger, les parents ou la force publique, développent ce que Birama Seck appelle « une surdi-mutité, voire une quasi-complicité envers la violence faite aux enfants» (Seck 2000 : 217). Pourquoi l'environnement social immédiat ne réagit-il pas? pourquoi garde-il secret de toutes ces exactions contre ses propres enfants ? C'est la question de fond à laquelle nous devons chercher à répondre.

24 À Lubumbashi, quand un enfant de la rue est auteur d'un forfait, le corps social admet comme normal le fait que la victime essaie de se rendre justice par elle-même. Ainsi J'ai assisté à une scène ou un enfant se faisait lentement écraser les doigts par un vendeur 
qui prétendait que l'enfant lui avait volé la somme de 500 francs. Personne ne s'attend à ce que quiconque dénonce la maltraitance qui est infligée à l'enfant. Ceci est manifeste dans le fait que, bien souvent, l'entourage immédiat de l'enfant est enclin à aider ou soutenir le «justicier» dans son action. Ainsi, quand un enfant de la rue est frappé, les passants dans une indifférence absolue ricanent et certains encouragent les persécuteurs en des termes comme : Pika nyoka ou «frappe le serpent »! À Mbuji Mayi, un groupe d'enfants de la rue avait été brûlé vif par des chercheurs de diamant pour avoir volé. Pareil traitement, montre que la norme sociale effective est extrêmement violente. Il n'est pas du tout sûr que ces enfants soient considérés comme des êtres humains à part entière. Perçu comme "sorciers" (symbole par excellence de la monstruosité anti-sociale) ou comme "serpents", ils ne méritent que mauvais traitement. Aucune protection ne leur est consentie, personne n'envisage de freiner leur consommation de produits psychotropes par exemple. L'enquête a même révélé que les enfants de la rue s'approvisionnaient en chanvre auprès de certains policiers et militaires! Ces produits psychotropes "sont achetés dans les différents kiosques pharmaceutiques qui pullulent à travers la ville. Ainsi, certains enfants de la rue, pour influer sur le fonctionnement de leur système nerveux, consomment plus de 20 comprimés ». (Kahola Tabu 2005 : 106).

Quelle interprétation les enfants de la rue se font-ils de leur maltraitance ? Andrea Rea remarque que «l'interprétation que les victimes donnent de leur vécu n'est donc pas uniforme et comme pour d'autres formes de présentation de soi, le récit de victimisation est l'occasion d'une mise en scène globale du social dont l'interprétation nécessite de tenir compte de la structure des transformations de la morphologie sociale » (Rea 1999: 28). De son côté, Inès Angelo souligne que « l'enfant a besoin de posséder un espace protégé au sein duquel il se sent à l'abri du regard ou du jugement d'autrui. L'absence d'un tel lieu est significative des difficultés à attribuer à l'enfant un espace psychique sans lequel il ne peut se reconnaître et s'autonomiser progressivement " (Angelo 2004: 155). Les enfants dits «de la rue " sont le triste résultat de la désorganisation de la structure sociale. Bon nombre de ces enfants viennent d'un espace familial qui ne le protégeait plus et les menaçaient. Ils y étaient régulièrement victimes de maltraitance physique ou psychique de la part de proches parents. Dans pareil contexte, les enfants finissent par opter pour la rue, espérant y trouver de meilleures conditions de vie. C'est l'ironie du sort, car les sévices corporels et psychiques qu'ils endurent dans la rue sont insoupçonnés de la société. Dès leur insertion dans la rue, ils sont plongés dans une autre violence. Ils apprennent alors à s'immuniser contre la douleur, à subir la violence mais aussi à la commettre. Tous ces actes de violence s'accomplissent dans l'indifférence totale de la société. Torturés et tortionnaires, violés et violeurs, telle est leur condition d'enfant de la rue. La relation entre les enfants de la rue et la société globale semble s'est installée dans des rapports de violence réciproque. Il est patent que la souffrance des enfants de la rue n'intéresse ni ne préoccupe la société qui reste prompte à les stigmatiser comme "sorciers", " démons ", " possédés ", " voleurs ", etc. Les enfants de la rue réagissent à cette mise au ban de la société par la violence et une agressivité permanentes contre les membres d'une société qui les abandonne à leur sort. 


\section{BIBLIOGRAPHIE}

Andrea Rea, 1999, Désintégration sociale et affaiblissement de l'Etat in Yves Cartuyves et Philippe Mary ; L'Etat face à l'insécurité. Dérives politiques des années 90. Bruxelles, Labor.

Angelino Inès, 2004, L'enfant, la famille, la maltraitance. Paris, Dunod.

Birama Seck, 2000, Les mauvais traitements à l'enfant au sénégal. Etats des lieux, prise de conscience, solutions In Thérèse Agossou (Ed.), Regards d'Afrique sur la maltraitance. Paris, Karthala.

D'haeyer Aurore, 2004, Enfants sorciers, entre magie et misère. Paris, Labor.

Dominique Gauthier, 1994, L'enfant victime d'abus exuels, Paris, PUF.

Kahola Tabu O., 2005, Les reflets de la rue in Kaumba Lufunda (Ed.), Les enfants de la rue au Katanga. Rapport d'enquête effectuée durant la dixième session des travaux de l'observatoire, juin-octobre 2003.

Kahola Tabu O. et all., 2004, Les enfants des rues in Kaumba Lufunda (Ed.), Approches de la criminalité dans la ville de Lubumbashi, Rapport des recherches effectuées durant la neuvième session des travaux de l'Observatoire août 2003.

Mulumbwa Mutambwa, 2005, Formes des violences sexuelles, in Mulumbwa Mutambwa (Ed.), Abus et violences sexuels faits à l'enfant et à la femme au Katanga. Sites de Lubumbashi et du poste frontalier de Kasumbalesa. Rapport des recherches effectuées durant la treizième session des travaux de l'Observatoire, décembre 2004-février 2005.

Situations de risque et interventions institutionnelles au Pérou in Enfants travailleurs, enfances volées. Bruxelles, Colophon.

Riccardo Luccini, 1996, Sociologie de la survie : L'enfant dans la rue. Paris, PUF.

\section{NOTES}

1. Il convient de noter que les garçons tailladés à la lame de rasoir et les filles violées, au-delà des traumatismes psychiques engendrés par ces violences encourent des risques supplémentaires d'infection par le VIH/SIDA.

2. « la participation d'un enfant à des activités sexuelles qu'il n'est pas en mesure de comprendre, qui sont inappropriées à son âge et à son développement psycho-sexuel sont des abus sexuels ». (Gauthier $1994: 9$ ).

\section{RÉSUMÉS}

Considérés comme des sorciers (D’Hayer 2004), des personnes hors normes, les enfants de la rue ne reçoivent aucun égard de la part d'une société qui les ostracisent. Dans leur réclusion, ils 
endurent non seulement les rudes conditions de la vie dans la rue, mais ils subissent aussi et font subir à autrui des sévices corporels et psychiques graves. Dans cette relation de violenté et de violent, ces enfants semblent ne pas intéresser le corps social qui ne leur accorde que violence et mépris. On peut se demander pourquoi la société adopte une telle attitude envers ces enfants? Mais cette étude ne s'intéresse pas seulement aux abus et aux violences dont sont victimes les enfants de la rue. Elle cherche à essayer de mieux comprendre l'exclusion sociale qui caractérise leur vie et la passivité de la société face aux violences dont ils sont victimes et auteurs. Cet article a donc pour objet d'établir un état des lieux de la violence des interactions dans la rue et et de faire ressortir les représentations qui s'y rattachent.

Title : Exercicing violence and isolation of street children of Lubumbashi. Considered to be witches (D' Hayer 2004), uncommon people, street children do not receive any consideration from a society which ostracize them. In their reclusion, they endure not only the hard living conditions in the street, but they also undergo and subject others to serious physical and psychic abuses. In this relation between actor and victim of violence, these children do not seem to interest the society which reserve for them only violence and contempt. We can ask ourselves why society has adopted such an attitude towards these children? But this study is not interested only in the abuses and violence of which the children of the street are victims. It rather seeks to understand better the social exclusion which characterizes their life and the passivity of society vis-a-vis violence of which they are victims and authors. The aim of this paper is thus to establish an inventory of the violence of interactions in the street and to emphasize the representations which are attached to it. 